DESY-09-213

\title{
The NMSSM and String Theory
}

\author{
Oleg Lebedev and Saúl Ramos-Sánchez \\ DESY Theory Group \\ Notkestrasse 85, D-22603 Hamburg, Germany
}

\begin{abstract}
We study the possibility of constructing the NMSSM from the heterotic string. String derived NMSSMs are much more rare than MSSMs due to the extra requirement that there exist a light singlet which couples to the Higgs pairs. They share the common feature that the singlet self-interactions are typically suppressed, leading to either the "decoupling" or the Peccei-Quinn limit of the NMSSM. In the latter case, the spectrum contains a light pseudoscalar which may be relevant to the MSSM fine-tuning problem. We provide a $\mathbb{Z}_{6}$ heterotic orbifold example of the NMSSM with approximate Peccei-Quinn symmetry, whose origin lies in the string selection rules combined with our choice of the vacuum configuration.
\end{abstract}




\section{Introduction}

The next-to-minimal supersymmetric Standard Model (NMSSM) is a minimal extension of the MSSM which includes a Standard Model (SM) singlet $S$ (for recent reviews, see $[1,2])$. It has certain advantages over the MSSM in that (i) it can provide a solution to the $\mu$-problem, (ii) it requires milder fine-tuning to accommodate the LEP Higgs bound. In the NMSSM, the SM-like Higgs boson $h$ can have unusual decay channels. If there is a light pseudoscalar $a, h$ can predominantly decay into pairs of $a$ 's, which subsequently decay into taus or light quarks [3]. For such final states, the LEP bound on the Higgs mass relaxes and can in some cases be as low as $90 \mathrm{GeV}$. As a result, the superpartners are not required to be very heavy for the Higgs mass to comply with the bound, and the fine-tuning problem of the MSSM can be avoided.

Motivated by these considerations, we undertake a search for string-derived NMSSMs. Recently, a number of different approaches have yielded examples of models with the exact MSSM spectrum. These include heterotic $\mathbb{Z}_{6}[4,5], \mathbb{Z}_{12}[6], \mathbb{Z}_{2} \times \mathbb{Z}_{2}[7]$ orbifolds as well as smooth Calabi-Yau compactifications [8-10] of the heterotic string 1 In fact, it has been shown in Refs. $[5,18]$ that there is a "fertile patch" in the heterotic landscape, where more than $0.1 \%$ of all inequivalent models have the MSSM spectrum. This result is based on the concept of "local GUTs" [4], which has been prompted by the orbifold GUT interpretation of the heterotic string models [19-21]. The main idea is that twisted matter comes from points in the compact space with $\mathrm{SO}(10)$ or $\mathrm{E}_{6}$ GUT symmetry and thus forms complete representations of the corresponding gauge group, while the (untwisted) gauge fields only respect the Standard Model symmetry in 4 dimensions. Many of the resulting models have a number of phenomenologically attractive features including R-parity [22], the neutrino seesaw [23] and preference for the TeV scale soft SUSY breaking masses [24].

In what follows, we explore the "fertile patch" (and beyond) of the heterotic minilandscape to identify the NMSSM candidates. Common features of these models are discussed in Section 2, while a specific example is presented in Section 3.

\section{Generalities}

The relevant superpotential is given by

$$
W=\lambda S H_{u} H_{d}+\frac{1}{3} \kappa S^{3},
$$

\footnotetext{
${ }^{1}$ This lists extends further if one allows for vector-like exotics [11-17].

${ }^{2}$ Some studies of the singlet extensions of the Pati-Salam model have been performed in [25].
} 
which corresponds to the " $\mathbb{Z}_{3}$-symmetric" NMSSM 3 . Here we are considering $S$ to be a "massless" at the string level singlet in the sense that its supersymmetric mass term is well below the electroweak scale. Furthermore, we are assuming that supersymmetry is not broken by the F-term of $S$ (in the limit $\langle S\rangle \rightarrow 0$ ) and thus the "tadpole" term linear in $S$ is also negligible. The corresponding soft supersymmetry breaking terms are

$$
-\mathcal{L}_{\text {soft }}=m_{S}^{2}|S|^{2}+\left(\lambda A_{\lambda} S H_{u} H_{d}+\frac{1}{3} \kappa A_{\kappa} S^{3}+\text { h.c. }\right)
$$

where we have omitted $S$-independent terms. In what follows, we will assume all soft terms to be of the same order of magnitude (EW size). We will also omit the $\mathrm{CP}$ - phases which are strongly constrained by electric dipole moments (see, e.g. [26]).

In general, $\lambda$ and $\kappa$ are the effective couplings,

$$
\lambda=\text { const }+\left\langle s_{a_{1}} \ldots s_{a_{n}}\right\rangle, \kappa=\left\langle s_{b_{1}} \ldots s_{b_{n}}\right\rangle,
$$

where $s_{i}$ are Standard Model singlets which get non-negligible VEVs in Planck units and the "const" indicates a direct trilinear coupling. The Standard Model singlet $S$ comes originally from the $\mathrm{E}_{8} \times \mathrm{E}_{8}$ sector of the theory and therefore carries charges under some of the gauge groups. The $S^{3}$ interaction violates these symmetries and only after gauge symmetries get broken spontaneously, is this effective interaction allowed. As a result, $\kappa$ is suppressed by the SM singlet VEVs $\left\langle s_{b_{1}} \ldots s_{b_{n}}\right\rangle$ (If $S$ comes from the gravitational sector, it is neutral under gauge symmetries but its interactions are suppressed). In contrast, a coupling among three different fields is allowed already at the trilinear level, hence the "const" term in Eq.(3).

The size of $\left\langle s_{i}\right\rangle$ is model-dependent. Some SM singlets are required to attain VEVs by supersymmetry. In particular, the presence of an anomalous $U(1)$ symmetry induces the Fayet-Iliopoulos (FI) term [27]

$$
D_{\mathrm{FI}}=\frac{g M_{\mathrm{Pl}}^{2}}{192 \pi^{2}} \operatorname{Tr} \mathrm{U}(1)_{\mathrm{anom}}+\sum_{i} q^{i}\left|s_{i}\right|^{2},
$$

which must vanish in a supersymmetric configuration. Here $q^{i}$ are the anomalous $\mathrm{U}(1)$ charges of $s_{i}$ and $g$ is the gauge coupling. Since $\operatorname{Tr} \mathrm{U}(1)_{\text {anom }} \neq 0$, some singlets develop VEVs somewhat below the Planck scale. This sets the scale for other singlet VEVs as well and one generally expects $\left\langle s_{i}\right\rangle$ to be in the range $\mathcal{O}\left(10^{-1}\right)-\mathcal{O}\left(10^{-2}\right)$ in Planck units, although $\mathcal{O}(1)$ VEVs are also possible.

\footnotetext{
${ }^{3}$ This symmetry is only approximate. For example, it can be broken by a (small) supersymmetric mass term for $S$.

${ }^{4}$ There is a caveat here: the massless singlet can be a linear combination of the type $N=\left(a S_{1}+\right.$ $\left.b S_{2}\right) / \sqrt{a^{2}+b^{2}}$, where $a, b$ are proportional to some VEVs. In this case, unsuppressed $N^{3}$-interaction may be allowed, although it is singular in the limit of vanishing VEVs. We find that in practice it does not happen since the string selection rules are very constraining.
} 
Therefore, typically

$\kappa \ll 1$,

while $\lambda$ can be order one. If the "const" term in Eq.(3) vanishes due to string selection rules, then $\lambda$ is also suppressed. We thus are led to two distinct versions of the NMSSM: the "decoupling" $(\lambda, \kappa \ll 1)$ and the Peccei-Quinn scenarios $(\kappa \ll 1)$. Let us consider these limits in more detail, following Ref. [1].

1) Decoupling limit. For $\lambda, \kappa \ll 1$, the singlet essentially decouples and the NMSSM degenerates into a version of the MSSM, albeit with modifications in the neutralino sector. The potential for (the real part of) the scalar component of $S$, denoted by $s$, is given by

$$
V(s) \sim m_{S}^{2} s^{2}+\frac{2}{3} \kappa A_{\kappa} s^{3}+\kappa^{2} s^{4} .
$$

For $A_{\kappa}^{2} \geq 8 m_{S}^{2}$, there is a local minimum at

$$
s \simeq \frac{1}{4 \kappa}\left(-A_{\kappa}+\sqrt{A_{\kappa}^{2}-8 m_{S}^{2}}\right) .
$$

Since the chargino mass bound requires $\lambda s \sim$ EW, we have

$$
s \sim \frac{\mathrm{EW}}{\kappa} \sim \frac{\mathrm{EW}}{\lambda}
$$

for the soft terms of the electroweak size. This defines the decoupling limit. The difference from the MSSM resides in the neutralino sector: the fermionic component of $S$ has mass $2 \kappa s$ and can be the LSP. The NLSP decays are then suppressed by the small coupling $\lambda$ leading to its long lifetime with characteristic signatures such as displaced vertices [28].

2) Peccei-Quinn limit. For $\kappa \ll 1$ [29], the model possesses an approximate PecceiQuinn symmetry $H_{u, d} \rightarrow e^{i \alpha} H_{u, d}, S \rightarrow e^{-2 i \alpha} S$. Spontaneous breaking of this symmetry generates a pseudo-Goldstone boson (axion). The composition of this state is given by

$$
\begin{aligned}
& A_{\mathrm{PQ}}=\frac{1}{\sqrt{v^{2} \sin ^{2} 2 \beta+4 s^{2}}}\left(v \sin 2 \beta A-2 s S_{I}\right), \\
& A=\cos \beta H_{u I}+\sin \beta H_{d I},
\end{aligned}
$$

where $S_{I}, H_{u I}, H_{d I}$ are defined by $f_{I} \equiv \sqrt{2} \operatorname{Im}(f-\langle f\rangle)$. As usual, $\tan \beta=v_{u} / v_{d}$ and $v=\sqrt{v_{u}^{2}+v_{d}^{2}}=174 \mathrm{GeV}$.

The presence of a light axion-like state can be relevant to the MSSM fine-tuning problem [3]. Typically, $s \gg v \sin 2 \beta$, so that the axion is predominantly an EW singlet. Its couplings to quarks and gauge bosons are suppressed, but the coupling to the Higgs bosons is significant. Thus the SM-like Higgs $h$ can decay into pairs of $A_{\mathrm{PQ}}$ which would subsequently decay into 4 fermions. If $m_{A_{P Q}}<2 m_{b}$, the dominant decay channel would 
be $h \rightarrow 2 A_{\mathrm{PQ}} \rightarrow 4 \tau(4 q)$, with $q$ being light quarks. Under these conditions, the LEP bound on the Higgs mass relaxes to about $105 \mathrm{GeV}$ for the final state taus and $90 \mathrm{GeV}$ for the final state light quarks [32]. This ameliorates the MSSM fine-tuning problem since the superpartners are not required to be very heavy to accommodate the LEP Higgs bound.

Let us conclude this section by noting that the above symmetries may appear puzzling from the low energy perspective. They are consequences of the stringy UV completion of these effective theories. For example, the absence or suppression of the $S^{3}$ term has to do with the fact that $S$ is charged under additional gauge symmetries. Similarly, the absence of the direct $\mu$-term is a result of the string selection rules and our choice of the vacuum state. It is interesting that these string constructions favor certain versions of the NMSSM.

\section{Search for the NMSSM}

The $\mathbb{Z}_{6}$-II heterotic orbifold is known to yield many examples of models with the MSSM spectrum. Particularly favorable are the gauge embeddings which produce local GUTs like $\mathrm{SO}(10)$ or $\mathrm{E}_{6}$ at some fixed points. Properties of these models are summarized in Refs. $[5,18]$. Clearly, to obtain an example of the NMSSM, one needs to impose the extra requirement that there exist at least one massless singlet which couples to the Higgs pair. This condition turns out to be very restrictive. In particular, we have analyzed the "fertile patch" of the mini-landscape with SO(10) local GUTs of Ref. [5] and found no NMSSM examples. These models contain 2 Wilson lines and appear to be quite rigid in the sense that the decoupling of exotics implies that all the SM singlets are also heavy 6 . In setups with 3 Wilson lines of Ref. [18], this is not the case and we have identified a number of the NMSSM candidates.

\subsection{Example: NMSSM with approximate Peccei-Quinn symmetry}

Here we present an example of a string model which matches closely properties of the NMSSM in the Peccei-Quinn limit. It is based on a heterotic orbifold $\mathbb{Z}_{6}$-II. In the notation of Ref. [18], the model is defined by the following gauge shift and Wilson lines (in the $\mathrm{E}_{8} \times \mathrm{E}_{8}$ root basis):

$$
\begin{aligned}
V & =\left(\frac{1}{6},-\frac{1}{3},-\frac{1}{2}, 0,0,0,0,0\right)(0,0,0,0,0,0,0,0), \\
W_{2} & =\left(1, \frac{1}{2}, 0, \frac{1}{2}, \frac{1}{2},-\frac{1}{2},-1,0\right)\left(-\frac{1}{4}, \frac{3}{4}, \frac{1}{4}, \frac{1}{4}, \frac{3}{4},-\frac{3}{4},-\frac{3}{4}, \frac{3}{4}\right), \\
W_{2}^{\prime} & =\left(\frac{3}{4}, \frac{3}{4},-\frac{1}{4},-\frac{1}{4},-\frac{1}{4}, \frac{3}{4}, \frac{1}{4}, \frac{1}{4}\right)\left(-\frac{1}{4},-\frac{1}{4},-\frac{1}{4},-\frac{1}{4},-\frac{1}{4}, \frac{1}{4}, \frac{1}{4}, \frac{3}{4}\right),
\end{aligned}
$$

\footnotetext{
${ }^{5}$ There are further constraints on this scenario from meson decays [30,31].

${ }^{6}$ This does not always apply to models with $\mathrm{E}_{6}$ local GUTS and we have identified one example leading to the NMSSM in the "decoupling" limit [33].
} 


$$
W_{3}=\left(-\frac{5}{6},-\frac{7}{6}, \frac{1}{2}, \frac{1}{2}, \frac{1}{2},-\frac{1}{2},-\frac{1}{2},-\frac{1}{2}\right)\left(0,0, \frac{1}{3}, \frac{1}{3}, \frac{1}{3}, 0,1, \frac{2}{3}\right) .
$$

The gauge group after compactification is

$$
G_{\mathrm{SM}} \times[\mathrm{SU}(6)] \times \mathrm{U}(1)^{7}
$$

where $G_{\mathrm{SM}}=\mathrm{SU}(3)_{C} \times \mathrm{SU}(2)_{\mathrm{L}} \times \mathrm{U}(1)_{Y}$ includes the standard $\mathrm{SU}(5)$ hypercharge generator

$$
\mathrm{t}_{Y}=\left(0,0,0, \frac{1}{3}, \frac{1}{3}, \frac{1}{3},-\frac{1}{2},-\frac{1}{2}\right)(0,0,0,0,0,0,0,0) .
$$

(Here we do not require the existence of non-anomalous $B-L$ symmetry.) The resulting massless spectrum is displayed in Table 1, At this step, it contains 3 SM families plus vector-like matter. One of the SM generations comes from the $\mathbf{2 7 - p l e t ~ o f ~} \mathrm{E}_{6}$ located at the fixed point at the origin in the compact space, while the other two come from

\begin{tabular}{|c|c|c|c|c|c|c|c|c|}
\hline$\#$ & Irrep & Label & $\#$ & Anti-irrep & Label & $\#$ & Irrep & Label \\
\hline 4 & $(\mathbf{3}, \mathbf{2} ; \mathbf{1})_{1 / 6}$ & $q_{i}$ & 1 & $(\overline{\mathbf{3}}, \mathbf{2} ; \mathbf{1})_{-1 / 6}$ & $\bar{q}_{i}$ & 63 & $(\mathbf{1}, \mathbf{1} ; \mathbf{1})_{0}$ & $s_{i}^{0}$ \\
\hline 9 & $(\mathbf{1}, \mathbf{2} ; \mathbf{1})_{-1 / 2}$ & $\ell_{i}$ & 6 & $(\mathbf{1}, \mathbf{2} ; \mathbf{1})_{1 / 2}$ & $\bar{\ell}_{i}$ & 4 & $(\mathbf{1}, \mathbf{1} ; \overline{\mathbf{6}})_{0}$ & $\bar{h}_{i}$ \\
\hline 4 & $(\overline{\mathbf{3}}, \mathbf{1} ; \mathbf{1})_{-2 / 3}$ & $\bar{u}_{i}$ & 1 & $(\mathbf{3}, \mathbf{1} ; \mathbf{1})_{2 / 3}$ & $u_{i}$ & 4 & $(\mathbf{1}, \mathbf{1} ; \mathbf{6})_{0}$ & $h_{i}$ \\
\hline 4 & $(\mathbf{1}, \mathbf{1} ; \mathbf{1})_{1}$ & $\bar{e}_{i}$ & 1 & $(\mathbf{1}, \mathbf{1} ; \mathbf{1})_{-1}$ & $e_{i}$ & & & \\
\hline 8 & $(\overline{\mathbf{3}}, \mathbf{1} ; \mathbf{1})_{1 / 3}$ & $\bar{d}_{i}$ & 5 & $(\mathbf{3}, \mathbf{1} ; \mathbf{1})_{-1 / 3}$ & $d_{i}$ & & & \\
\hline 1 & $(\mathbf{3}, \mathbf{1} ; \mathbf{1})_{1 / 6}$ & $v_{i}$ & 1 & $(\overline{\mathbf{3}}, \mathbf{1} ; \mathbf{1})_{-1 / 6}$ & $\bar{v}_{i}$ & & & \\
\hline 1 & $(\mathbf{1}, \mathbf{1} ; \overline{\mathbf{6}})_{1 / 2}$ & $w_{i}^{+}$ & 1 & $(\mathbf{1}, \mathbf{1} ; \mathbf{6})_{-1 / 2}$ & $w_{i}^{-}$ & & & \\
\hline 9 & $(\mathbf{1}, \mathbf{1} ; \mathbf{1})_{1 / 2}$ & $s_{i}^{+}$ & 9 & $(\mathbf{1}, \mathbf{1} ; \mathbf{1})_{-1 / 2}$ & $s_{i}^{-}$ & & & \\
\hline 6 & $(\mathbf{1}, \mathbf{2} ; \mathbf{1})_{0}$ & $m_{i}$ & & & & & & \\
\hline
\end{tabular}
various twisted and untwisted sectors. All three generations are intrinsically different in this model. Further details can be found in [33].

Table 1: Massless spectrum. Representations with respect to $\left[\mathrm{SU}(3)_{C} \times \mathrm{SU}(2)_{\mathrm{L}}\right] \times$ $[\mathrm{SU}(6)]$ are given in bold face, the hypercharge is indicated by the subscript.

At the next step, many of the SM singlets develop VEVs and break the gauge group to

$$
G_{\mathrm{SM}} \times[\mathrm{SU}(6) \times \mathrm{U}(1)]
$$

where $[\mathrm{SU}(6) \times \mathrm{U}(1)]$ is hidden in the sense that no $\mathrm{SM}$ particle is charged under this group. At the same time, the unwanted vector-like exotics attain large masses and decouple. The resulting massless spectrum is that of the MSSM plus, possibly, SM singlets.

We choose a specific configuration of the SM singlet VEVs, in which only the fields

$$
\left\{\widetilde{s}_{i}\right\}=\left\{s_{1}^{0}, s_{2}^{0}, s_{7}^{0}, s_{12}^{0}, s_{14}^{0}, s_{21}^{0}, s_{22}^{0}, s_{27}^{0}, s_{30}^{0}, s_{31}^{0}, s_{35}^{0}, s_{37}^{0}, s_{40}^{0}, s_{41}^{0}, s_{44}^{0}, s_{45}^{0}, s_{61}^{0}, s_{63}^{0}\right\}
$$


develop non-zero VEVs, while the expectation values of all other fields vanish. We have checked that, in this case, the mass matrices for the exotics have maximal rank. The cancellation of the Fayet-Iliopoulos term and D-flatness are guaranteed by the holomorphic monomial

$$
\psi=\left(s_{1}^{0}\right)^{7}\left(s_{2}^{0}\right)^{5}\left(s_{7}^{0}\right)^{2}\left(s_{12}^{0}\right)^{2}\left(s_{14}^{0}\right)^{4} s_{21}^{0} s_{22}^{0} s_{27}^{0}\left(s_{30}^{0}\right)^{2}\left(s_{31}^{0}\right)^{2} s_{35}^{0} s_{37}^{0} s_{40}^{0} s_{41}^{0} s_{44}^{0} s_{45}^{0}\left(s_{61}^{0}\right)^{6} s_{63}^{0} .
$$

The massless (to order 6 in singlet VEVs) pair of Higgses is given schematically by

$$
\begin{aligned}
& H_{u} \sim \bar{\ell}_{1}+\widetilde{s} \bar{\ell}_{2}+\bar{\ell}_{3}+\bar{\ell}_{4}+\bar{\ell}_{5}, \\
& H_{d} \sim \ell_{9},
\end{aligned}
$$

where $\ell_{i}, \bar{\ell}_{i}$ are the SU(2) doublets of Table 1 and we have omitted order 1 coefficients. There exists one massless SM singlet

$$
S=s_{66}^{0}
$$

and it couples to the Higgs pair at the trilinear order:

$$
\lambda S H_{u} H_{d}=\lambda s_{66}^{0} \bar{\ell}_{3} \ell_{9}+\text { higher order terms } .
$$

This is a twisted coupling of the type $T_{5} T_{5} T_{2}$, where $T_{i}$ denotes a twisted sector. On the other hand, the self-interaction $S^{3}$ is not allowed at least to order $\tilde{s}^{5}$. Thus, in terms of Eq.(11), we have

$$
\begin{aligned}
& \lambda \sim 1, \\
& \kappa<\mathcal{O}\left(\tilde{s}^{5}\right) .
\end{aligned}
$$

For $\widetilde{s}<1$, the system has an approximate Peccei-Quinn symmetry, whose spontaneous breaking results in a light pseudoscalar state $A_{\mathrm{PQ}}$. Its mass depends on the order of the allowed coupling as well as the exact value of $\tilde{s}$ and can be light enough to be relevant to the MSSM fine-tuning problem.

Let us also note that the discrete symmetry of this $\mathbb{Z}_{3}-$ NMSSM is expected to be broken by a small supersymmetric mass term for the singlet, which helps avoid cosmological problems associated with spontaneous breaking of discrete symmetries.

The existence of approximate symmetries in the low-energy theory is a result of the string selection rules combined with a specific choice of the vacuum configuration (14). From the bottom-up perspective, it is not transparent why the term $S^{3}$ for an SM singlet is not allowed. This becomes clear in the UV completion of the model: $S$ stems from the $\mathrm{E}_{8} \times \mathrm{E}_{8}$ sector and thus carries additional gauge charges. Similarly, the bare $\mu-$ term is not allowed (to order $\tilde{s}^{6}$ ) due to our choice of the vacuum configuration and the string selection rules for the couplings. Thus, the approximate Peccei-Quinn symmetry is enforced by (14). Similarly, an example of approximate R-symmetry was constructed in Ref. [34]. 


\section{Conclusion}

We have undertaken a search for the NMSSM in the framework of the heterotic string compactified on a $\mathbb{Z}_{6}$-II orbifold. Although there are many models with the MSSM spectrum, the NMSSM-like models are rare. This is due to the additional requirement that there exist at least one light SM singlet that couples to the Higgs pair. Our search within the "fertile patch" of the heterotic landscape with $\mathrm{SO}(10)$ local GUTs [5] has given null results, yet we have found a number of the NMSSM candidates in setups with 3 Wilson lines $[18]$.

The stringy NMSSMs share the common feature that the singlet self-interactions are typically suppressed, which leads to specific versions of the NMSSM. In one variant, the singlet sector essentially decouples from the MSSM, while in the other there is an approximate Peccei-Quinn symmetry whose breaking leads to a light pseudoscalar. The latter can be relevant to the MSSM fine-tuning problem since in this case the bound on the Higgs mass relaxes.

The apparent (approximate) symmetries of the low energy theory result from properties of its stringy UV completion. For instance, the suppression of the direct $\mu$-term and the singlet self-interactions is due to the string selection rules combined with our choice of the vacuum state. The ensuing Peccei-Quinn symmetry is broken by higher order terms in the superpotential.

\section{References}

[1] U. Ellwanger, C. Hugonie and A. M. Teixeira, arXiv:0910.1785 [hep-ph].

[2] M. Maniatis, arXiv:0906.0777 [hep-ph].

[3] R. Dermíšek and J. F. Gunion, Phys. Rev. Lett. 95, 041801 (2005); R. Dermíšek, Mod. Phys. Lett. A 24, 1631 (2009).

[4] W. Buchmüller, K. Hamaguchi, O. Lebedev and M. Ratz, Phys. Rev. Lett. 96, 121602 (2006); Nucl. Phys. B 785, 149 (2007).

[5] O. Lebedev, H. P. Nilles, S. Raby, S. Ramos-Sánchez, M. Ratz, P. K. S. Vaudrevange and A. Wingerter, Phys. Lett. B 645, 88 (2007).

[6] J. E. Kim and B. Kyae, arXiv:hep-th/0608085.

[7] M. Blaszczyk, S. G. Nibbelink, M. Ratz, F. Ruehle, M. Trapletti and P. K. S. Vaudrevange, arXiv:0911.4905 [hep-th]; see also G. B. Cleaver, A. E. Faraggi and D. V. Nanopoulos, Phys. Lett. B 455, 135 (1999).

[8] V. Bouchard and R. Donagi, Phys. Lett. B 633, 783 (2006). 
[9] V. Braun, Y. H. He, B. A. Ovrut and T. Pantev, JHEP 0605, 043 (2006).

[10] L. B. Anderson, J. Gray, Y. H. He and A. Lukas, arXiv:0911.1569 [hep-th].

[11] L. E. Ibáñez, J. E. Kim, H. P. Nilles and F. Quevedo, Phys. Lett. B 191, 282 (1987).

[12] A. Font, L. E. Ibáñez, H. P. Nilles and F. Quevedo, Phys. Lett. 210B, 101 (1988) [Erratum-ibid. B 213, 564 (1988)].

[13] J. A. Casas and C. Muñoz, Phys. Lett. B 209, 214 (1988); Phys. Lett. B 214, 63 (1988).

[14] T. P. T. Dijkstra, L. R. Huiszoon and A. N. Schellekens, Nucl. Phys. B 710, 3 (2005).

[15] R. Blumenhagen, S. Moster and T. Weigand, Nucl. Phys. B 751, 186 (2006).

[16] A. E. Faraggi, C. Kounnas and J. Rizos, Phys. Lett. B 648, 84 (2007).

[17] F. Gmeiner and G. Honecker, JHEP 0807, 052 (2008).

[18] O. Lebedev, H. P. Nilles, S. Ramos-Sánchez, M. Ratz and P. K. S. Vaudrevange, Phys. Lett. B 668 (2008) 331.

[19] T. Kobayashi, S. Raby and R. J. Zhang, Phys. Lett. B 593, 262 (2004); Nucl. Phys. B 704, 3 (2005).

[20] S. Förste, H. P. Nilles, P. K. S. Vaudrevange and A. Wingerter, Phys. Rev. D 70, 106008 (2004).

[21] W. Buchmüller, K. Hamaguchi, O. Lebedev and M. Ratz, Nucl. Phys. B 712, 139 (2005). See also W. Buchmüller, C. Lüdeling and J. Schmidt, JHEP 0709, 113 (2007).

[22] O. Lebedev, H. P. Nilles, S. Raby, S. Ramos-Sánchez, M. Ratz, P. K. S. Vaudrevange and A. Wingerter, Phys. Rev. D 77, 046013 (2008).

[23] W. Buchmüller, K. Hamaguchi, O. Lebedev, S. Ramos-Sánchez and M. Ratz, Phys. Rev. Lett. 99, 021601 (2007).

[24] O. Lebedev, H. P. Nilles, S. Raby, S. Ramos-Sánchez, M. Ratz, P. K. S. Vaudrevange and A. Wingerter, Phys. Rev. Lett. 98, 181602 (2007).

[25] M. Chemtob and P. Hosteins, arXiv:0909.4497 [hep-ph].

[26] S. Abel, S. Khalil and O. Lebedev, Nucl. Phys. B 606, 151 (2001); Phys. Rev. Lett. 89, 121601 (2002).

[27] M. Dine, N. Seiberg and E. Witten, Nucl. Phys. B 289, 589 (1987). 
[28] U. Ellwanger and C. Hugonie, Eur. Phys. J. C 5, 723 (1998).

[29] D. J. Miller, R. Nevzorov and P. M. Zerwas, Nucl. Phys. B 681, 3 (2004).

[30] R. Dermíšek, J. F. Gunion and B. McElrath, Phys. Rev. D 76, 051105 (2007).

[31] F. Domingo, U. Ellwanger, E. Fullana, C. Hugonie and M. A. Sanchis-Lozano, JHEP 0901, 061 (2009).

[32] A recent update of the Higgs mass bound can be found in "20 years of Aleph data", CERN, http://indico.cern.ch/conferenceDisplay.py?confId=71475

[33] O. Lebedev and S. Ramos-Sánchez, Orbifold tables, 2009, http://www.th.physik.unibonn.de/nilles/Z6IIorbifold/nmssm/.

[34] R. Kappl, H. P. Nilles, S. Ramos-Sánchez, M. Ratz, K. Schmidt-Hoberg and P. K. S. Vaudrevange, Phys. Rev. Lett. 102, 121602 (2009). 